\section{Survival of extended-spectrum beta-lactamase producing escherichia coli in dog faeces}

\author{
Sasha-Anne Senior ${ }^{1}$, Amy L. Wedley ${ }^{2}$, \\ Nicola J. Williams ${ }^{2}$, Thomas W. Maddox ${ }^{3}$ \\ 1 School of Veterinary Science, University of Liverpool, Liverpool, \\ Merseyside L69 3BX, UK \\ 2 National Consortium for Zoonosis Research, School of Veterinary \\ Science, University of Liverpool, Neston, CH64 7TE, UK \\ 3 Department of Musculoskeletal Biology, Institute of Aging and \\ Chronic Disease, University of Liverpool, Neston, CH64 7TE, UK
}

\begin{abstract}
AIMS OF STUDY
The increasing prevalence of antimicrobial-resistance amongst bacteria poses significant problems for human and veterinary medicine. Production of extended-spectrum beta-lactamases (ESBLs) by bacteria such as Escherichia coli represents a particular concern; these enzymes confer resistance to a wide range of beta-lactam antimicrobials, including the critically important third-generation cephalosporins. Faeces from dogs with resistant bacteria may disseminate, transmit or propagate antimicrobial resistance. However, it is unknown how long such bacteria can survive in canine faeces and hence their risk in perpetuating antimicrobial resistance is uncertain. The aims of this study were to determine the survival numbers and times of ESBL-producing $E$. coli in dog faeces maintained at different temperatures and conditions, and to evaluate if survival of ESBL-producing $E$. coli differs from non-ESBL-producing, multidrug resistant (MDR) strains.
\end{abstract}

\section{METHODS}

Freshly collected canine faecal samples were determined to be free of ESBL-producing and MDR E. coli. These were then separately inoculated with approximately $10^{6}$ CFU (colony forming units)/g of two previously obtained MDR and ESBLproducing $E$. coli strains retrieved from an archived culture collection. Samples were then stored in open plastic containers under three conditions: i) incubated at $23^{\circ} \mathrm{C}$, ii) cold storage at $4^{\circ} \mathrm{C}$ and iii) outside under variable temperatures. Samples were removed at 0, 1, 3, 5, 7, 14 and 21 days for enumeration of bacterial numbers on eosin methylene blue agar (EMBA) containing relevant screening antimicrobials. Recovered isolates were confirmed as $E$. coli with the $E$. coli specific uidA PCR and tested for ESBL-production and multidrug resistance. Survival curves were plotted, with regression analysis performed on the linearized survival slopes and comparison of slopes by analysis of covariance (ANCOVA).

\section{RESULTS}

All bacteria survived under all conditions for a minimum of 7 days, with both ESBL-producing and MDR E.coli surviving in high numbers (105-106 CFU/g) for at least 21 days under incubator and cold storage conditions. Bacteria were not detectable under outside conditions after 14 days for MDR $E$. coli and after 7 days for ESBL-producing E. coli. There was no significant difference in survival slopes between the two bacterial strains for each of the three conditions. However, there was a significant difference in the survival slopes for both ESBL-producing E.coli and MDR E. coli between the different conditions ( $P=0.002$ and $P=0.03$ respectively).

\section{CONCLUSIONS}

These results demonstrate that survival of relatively high numbers of multidrug resistant and ESBL-producing $E$. coli is possible in canine faeces for moderately extended periods. There does not seem to be a significant difference in survival slopes between ESBL-producing and non-ESBLproducing multidrug resistant $E$. coli strains, but for both strains survival is decreased under outside conditions.

Faeces of dogs carrying antimicrobial-resistant bacteria may have role to play in maintenance of antimicrobial resistance and appropriate handling of canine faeces is important to limit the dissemination of such bacteria.

All work supported by BSAVA PetSavers Grant.

\section{The influence of androgen} receptor polymorphisms on the development of cruciate disease in Rottweilers

\section{Madeleine Ovenden, Siobhan Simpson, Raushan Jaffar, Harriet Will, Shareen Aktar. Catrin Rutland, Malcolm Cobb, Mark Dunning, Nigel Mongan.}

School of Veterinary Medicine and Science, University of Nottingham, Sutton Bonington Campus, Leicestershire, LE12 5RD, UK

Cruciate ligament disease (CLD) is commonly cited as one of the most common orthopaedic problems in dogs and the last few years has seen a shift in epidemiology of the disease from older mixed breeds dogs to young, large breed dogs such as the Rottweiler. Androgens are key influences on the rapid growth seen in large breed dogs and polymorphic regions in the canine androgen receptor have been linked with a number of diseases. This study investigated whether polymorphic regions in the canine androgen receptor influenced the development of CLD in 423 Rottweilers. The study also gathered epidemiological information about the 423 Rottweilers by questionnaire. There was no significant difference in length of androgen receptor and development of CLD in all dogs except female neutered dogs where there was a significant result $(P=0.036)$. Analysis of questionnaire data also found that neutering significantly increased the risk of dogs developing CLD ( $P=0.0002)$. No significance was found between CLD and epidemiological factors such as weight, exercise and diet. This suggests that genetic factors could be of greater importance than epidemiological factors in developing CLD and warrants further investigation in Rottweilers and other large breed dogs, in particular neutered females. 